\title{
Simulation of the hard-sphere crystal-melt interface
}

\author{
Ruslan L. Davidchack and Brian B. Laird \\ Department of Chemistry, University of Kansas, Lawrence, Kansas 66045
}

(Received 6 January 1998; accepted 4 March 1998)

\begin{abstract}
In this work, we examine in detail the structure and dynamics of the face-centered cubic (100) and (111) crystal-melt interfaces for systems consisting of approximately $10^{4}$ hard spheres using molecular dynamics simulation. A detailed analysis of the data is performed to calculate density, pressure, and stress profiles (on both fine and coarse scales), as well as profiles for the diffusion and orientational ordering. The strong dependence of the coarse-grained profiles on the averaging procedure is discussed. Calculations of 2-D density contours in the planes perpendicular to the interface show that the transition from crystal to fluid occurs over a relatively narrow region (over only 2-3 crystal planes) and that these interfacial planes consist of coexisting crystal- and fluidlike domains that are quite mobile on the time scale of the simulation. We also observe the creation and propagation of vacancies into the bulk crystal. (C) 1998 American Institute of Physics.
\end{abstract}

[S0021-9606(98)51222-6]

\section{INTRODUCTION}

Detailed knowledge of the microscopic structure, dynamics, and thermodynamics of the crystal-melt interface is central to the full understanding of such important phenomena as near-equilibrium crystal growth and homogeneous nucleation. ${ }^{1-3}$ Given the severe difficulties associated with the design of experiments that are able to probe the interface at an atomic length scale, ${ }^{5}$ the vast majority of studies on such systems have involved computer simulation of model systems (see the review by Laird and Haymet ${ }^{4}$ and references therein.) In addition to establishing the basic phenomenology, the computer simulations are important in providing data for the evaluation and testing of theories of interfacial structure. The most promising theories for the prediction of the detailed microscopic structure and thermodynamics of crystal-melt interfaces are those based on densityfunctional theory-for more details see the reviews by Singh, ${ }^{6}$ Löwen, ${ }^{7}$ and Laird and Haymet. ${ }^{4}$

In a simulation, the interface is characterized by measuring the change in the various structural, thermal, and dynamical quantities of interest as the interface is traversed from one phase to the other. For planar interfaces, the $z$ axis is generally chosen as the direction perpendicular to the interface and quantities are averaged over $x y$ and presented as functions of $z$. Examples include, the density profile $\rho(z)$ $=\langle\rho(\mathbf{r})\rangle_{x y}$, the diffusion profile $D(z)$, the temperature profile $T(z)$, and pressure tensor profile $P(z)$. Such profiles will average out any inhomogeneities in the planes parallel to the interface and, as these can be extremely interesting, it can also useful to study $x y$ contour plots of given quantities on a layer-by-layer basis. The thermodynamic quantity of greatest interest for a particular interface is the solid-liquid surface free energy, $\gamma_{\mathrm{sl}}$, which is defined as the work required to form one unit area of interface-this quantity is difficult to determine via simulation and only one reliable calculation has been done, namely, that of Broughton and Gilmer on a Lennard-Jones system. ${ }^{8}$
In this work, we examine in detail the structure and dynamics of the face-centered cubic (FCC) (100) and (111) crystal-melt interfaces for systems consisting of approximately $10^{4}$ hard spheres using molecular dynamics (MD) simulation. (For an excellent introduction to the technique of MD simulation as applied to hard-sphere systems, see Ref. 9.) Although it is only a cartoon of real interatomic interactions, the hard-sphere system is interesting for two reasons: First, due to the relative simplicity of the interaction potential (either $\infty$ when two particles overlap or 0 otherwise), this system lends itself well to theoretical study-most of the density-functional theory calculations of crystal-melt interfacial structure involve hard-sphere systems. Second, it is now well established that the structure and freezing behavior of dense, simple fluids is, for the most part, determined by the repulsive part of the interaction potential. The effect of the attractive part of the interaction can be well accounted for by treating it as a perturbation to the repulsive part of the potential, which is often approximated by a hard-sphere with an effective diameter. ${ }^{10}$ Thus the hard-sphere crystal-melt interface is an important reference system for the general study of interfaces of systems with more realistic interactions.

The interface between an FCC hard-sphere crystal and its melt has been the subject of two recent simulation studies. Kyrlidis and Brown $(\mathrm{KB})^{11}$ have performed Monte Carlo simulations for the (111), (110), and (100) interfaces. The number of particles was about 3000 for each interface studied and only the density profiles were calculated, since the primary goal of the simulations was to test the results for this quantity from various density-functional theories. The interfacial widths, based on the density profiles, were shown to be about $4-5 \sigma, 5-6 \sigma$, and $4 \sigma$, for the (100), (111), and (110) interfaces, respectively. An observation of particular interest is the increase in the density-profile peak spacing that occurs in the (100) interface as one moves perpendicular to the interface from solid to fluid-this expansion is not seen in the (111) density profile. The limiting value of the (100) peak 
spacing on the liquid side was found to be almost precisely the same as the (111) bulk crystal spacing. The question as to the origin of this expansion was left open by Kyrlidis and Brown and will be examined further in this work.

In another recent work, Mori, Manabe, and Nishioka $(\mathrm{MMN})^{12}$ used constant energy molecular-dynamics simulation to study the same hard-sphere interfaces, also using systems on the order of 3000 particles. As with the KB Monte Carlo simulations only the density profiles were calculated, giving estimated widths of about $5.5 \sigma$ for all interfaces studied (these widths were not given by MMN, but we estimated them from their published plots). The runs here were relatively short (about 700 collisions per particle, including equilibration time); consequently, the density profiles showed significant statistical fluctuations especially in the liquid phase where periodic oscillations are seen to extend throughout the bulk fluid region-these are probably undissipated remnants of the FCC structure from which the fluid was produced.

The purpose of the present work is to expand upon these earlier simulations through the use of larger systems, longer simulation runs and a more detailed analysis. In particular we perform a layer-by-layer analysis of the density contours and orientational order to determine the origin of the expansion of the lattice plane spacing in (100). We also study the effect of a variety of averaging techniques on the results obtained-for example, we see that when lattice plane expansion is present the use of a uniform bin spacing in the $z$ direction for coarse-grained averages gives significantly different results than when a nonuniform bin spacing, determined by the spacing of the density peaks, is used. The rest of the paper is organized as follows: In the next section, we discuss the method by which our interface is prepared and subsequently equilibrated. The procedure by which averages are taken is presented in Sec. III. The results for the (100) and (111) interfaces are presented in the next three sections: Structure (Sec. IV), Stress and Pressure Profiles (Sec. V), and Transport (Sec. VI). In Sec. VII we conclude.

\section{INTERFACE PREPARATION AND EQUILIBRATION}

The crystal-melt interfaces are set up parallel to the $x-y$ plane with periodic boundary conditions in all directions, but since the symmetry of the system is broken in the $z$ direction, we use a simulation box that is much longer in that direction than in $x$ or $y$. The periodic boundary conditions result in a simulation box containing two interfaces - the length of the system in the $z$ direction must be chosen to be large enough that bulk behavior obtains in the center of both the crystal and liquid regions.

The bulk density of the crystal and fluid phases were chosen starting from the coexistence values that were previously determined by Hoover and Ree ${ }^{13}$ be $\rho_{c} \sigma^{3}=1.041$ and $\rho_{f} \sigma^{3}=0.945$, where $\rho_{c}, \rho_{f}$ and are the coexistence values of the hard-sphere crystal and fluid, respectively, and $\sigma$ is the hard-sphere diameter. The stability of the interface is very sensitive to the exact values of the coexistence parameters and we found that it was necessary to modify the Hoover and Ree results slightly to guarantee a strain-free bulk crystal after equilibration. Our final values are $\rho_{c} \sigma^{3}=1.037$ and $\rho_{f} \sigma^{3}=0.938$, which gives an equilibrium pressure of 11.55 $\pm 0.05 k_{B} T / \sigma^{3}$, as opposed to the $11.7 k_{B} T / \sigma^{3}$ obtained from the Hoover and Ree values. (Note: We use $2 \sigma$ error bars for all reported results of this study.)

The (100) system was initialized with 42 crystal layers each containing 128 particles with the same number of particles in the fluid, giving 10752 particles and dimensions $L_{x}=L_{y}=12.55 \sigma$, and $L_{z}=69.28 \sigma$. The initial (111) system has 36 crystal layers containing 154 particles each with an equal number of particles in the fluid. The size of the (111) system is 11088 particles with dimensions $L_{x}=12.20 \sigma, L_{y}$ $=13.45 \sigma$, and $L_{z}=68.57 \sigma$.

Initially the fluid is prepared separately as a bulk system. To ensure a nonoverlapping initial configuration, the hard spheres are set up on an ordered lattice-in this case FCC. This system is then evolved until the initial order disappears and the equilibrium fluid state is reached. This procedure, however, is not optimal for a fluid with densities close to the coexistence value, since it takes a very long run for the initial order to disappear. Therefore, we first create a fluid phase at a density about $20 \%$ below coexistence and then gradually increase the sphere diameters until the desired density is reached. The diameter increase is usually done in $4-5$ cycles each consisting of three steps: first, the spheres are moved away from each other by a small distance using a short-range repulsive potential and a conjugate gradient method; second, the sphere diameters are increased by a small factor so that no overlap is caused; third, the fluid is allowed to equilibrate for a relatively short period of time. Such a procedure allows one to create a fluid at densities even higher than at coexistence.

After the fluid block is equilibrated at a density slightly above the coexistence value, it is placed next to the perfectly ordered crystal block with a small gap to prevent overlap. The fluid particles are then assigned velocities according to a Maxwell distribution and are allowed to move, while the particles in the crystal are held fixed. The fluid fills the gap and its average total density decreases to the coexistence value. The value of the original density of the bulk fluid is chosen so that after equilibration the transverse pressure on the solid and fluid sides of the interface are equal - the exact values of the coexisting fluid and solid bulk densities (and thus the coexistence pressure) are adjusted to ensure that, after a long run, the overall stress in the bulk solid is zero. If the coexistence densities are not quite correct, the crystal will try to compensate by expanding and contracting in the $z$ direction, but since the periodic boundaries prohibit expansion in the $x$ or $y$ directions, stress will build up in the crystal.

\section{COMPUTING AVERAGES}

The interface can be characterized by $z$ dependent profiles of a variety of averaged quantities, such as density, pressure tensor, temperature, etc. These $x-y$ averaged parameter profiles are generated by partitioning the $z$ axis into discrete bins. In order to study various properties of the interface, different bin sizes were utilized. For the coarse scale the width of the bins was set equal to the bulk crystal layer spacing, which was $0.7841 \sigma$ and $0.9054 \sigma$ for the (100) and 
(111) orientations, respectively. For the fine scale each coarse-scale bin was divided into 25 equal parts. In addition, a nonuniform coarse scale was employed in order to study changes on a per-layer basis of those parameters that exhibit oscillations in the crystal. The boundaries of the nonuniform coarse-scale bins were defined to be at the minima in the fine-scale density profile of the crystal and extended into the fluid with the spacing between the last two detectable minima. The coarse scales help to reveal the features in the structural and thermodynamic parameters of the interface which, on the fine scale, are masked by the large inhomogeneity of the crystal.

In the course of this study we have found that the coarsescale profiles are extremely sensitive to the choice of the binning process, often leading to incorrect conclusions about the appearance of the coarse-scale profiles. We have found that a more reliable coarsed-grained profile, which is independent of the binning process, can be obtained by filtering the fine-scale profiles using a finite impulse response (FIR) filter. ${ }^{14}$ Given a fine-scale profile $f_{n}$, the filtered profile is obtained as follows

$$
\bar{f}_{n}=\sum_{k=-N}^{N} w_{k} f_{n+k}
$$

Since we want the filtering process to smooth out the large oscillations in the fine-scale profiles, we find the filter coefficients $w_{k}$ by minimizing the quantity

$$
S=\sum_{n}\left(\delta^{2} \bar{f}_{n}\right)^{2},
$$

where $\delta^{2} \bar{f}_{n}=\bar{f}_{n+1}+\bar{f}_{n-1}-2 \bar{f}_{n}$ is the second central difference.

The order of the FIR filter $2 N+1$ has to be large enough so that the oscillations due to the layered structure of the crystal are averaged over, but sufficiently small to retain the essential features of the profile in the interfacial region. We have found that for $N>40$ most of the oscillations disappear and the filter coefficients $w_{k}$ assume a Gaussian-type shape. Therefore, in order to reduce the number of independent parameters, we define the shape of the filter to be a Gaussian

$$
w_{k}=A e^{-(k / \epsilon)^{2}}, \quad k=-N, \ldots, N,
$$

with $\epsilon$ chosen to minimize $S$ given by Eq. (2). The normalization constant $A$ is determined from the condition $\Sigma w_{k}$ $=1$. Eventually we have set $N=50$ for all the fine-scale profiles in both (100) and (111) orientations, which corresponds to the filter width equal to approximately four crystal layers. The Gaussian width $\epsilon$ did not vary significantly for different profiles and was found to fall within the range $\epsilon=22 \pm 1$.

Even though the total momentum of the system is set to zero in the reference frame of the simulation cell, a nonzero diffusion in the fluid part of the system causes fluctuations in the average positions of the crystal layers. If the bin positions were defined relative to the simulation cell, this would lead to artificial broadening of the crystal density peaks. Therefore, we fix the bin boundaries with respect to the average position of the crystal by monitoring the average displacements of particles in the crystal away from their equilibrium lattice sites, and translating the bins by the average displacement every 0.4 collisions per particle (cpp) (defined as twice the ratio of the number of collisions to the number of particles in the system). This procedure does not alter the dynamics of the system and it guarantees that the bulk crystal is stationary during the averaging process.

In order to monitor variations of the system parameters across the interface, different parameter profiles have been defined as follows:

(a) Density: The density profile is defined as

$\rho(z)=\frac{\left\langle N_{z}\right\rangle}{L_{x} L_{y} \Delta z}$,

where $N_{z}$ is the number of particles between $z-\Delta z / 2$ and $z+\Delta z / 2$ at time $t ; L_{x}, L_{y}$ are the $x$ and $y$ dimensions of the system, and the angled brackets denote time averaging. The density profile is studied on both fine and coarse scales. The fine-scale density profile is used to determine the nonuniform coarse-scale bins.

(b) Temperature: The temperature profile is defined as

$T(z)=\frac{m}{3 k_{B}} \frac{1}{L_{x} L_{y} \Delta z \rho(z)}\left\langle\sum_{i=1}^{N_{z}} v_{i}^{2}\right\rangle$,

where $v_{i}$ is the velocity of a particle between $z$ $-\Delta z / 2$ and $z+\Delta z / 2$ at time $t, m$ is the mass of the particles, and $k_{B}$ is the Boltzmann constant. Defined on a coarse scale, the temperature profiles shows initial heating of the interfacial region, which decays after $300-400$ cpp. ${ }^{15}$ Uniformity in the temperature profile indicates thermal equilibrium of the interfacial system.

(c) Pressure: The pressure tensor profile can be calculated as the sum of the ideal gas pressure and the excess pressure due to hard-sphere collisions

$P_{k l}(z)=\rho(z) k_{B} T(z) \delta_{k l}+P_{k l}^{\mathrm{ex}}(z)$,

where $\delta_{k l}$ is the Kronecker delta. The excess part can be determined based on the virial theorem as follows:

$P_{k l}^{\mathrm{ex}}(z)=\frac{m}{L_{x} L_{y} \Delta z \Delta t} \sum_{c=1}^{N_{c}} x_{k} \Delta v_{l}$,

where $\Delta t$ is the time interval, $N_{c}$ is the number of collisions that occur between $z-\Delta z / 2$ and $z+\Delta z / 2$ during that interval, $x_{k}$ are the $x, y$, or $z$ components of the relative distance between two colliding particles, and $\Delta v_{l}$ are the components of the velocity change due to collision. If the centers of the two particles are in different bins at the moment of collision, then half of the virial for this collision is assigned to each of the two bins. The total pressure profile is then given by one-third of the trace of the pressure tensor

$P(z)=\frac{1}{3}\left[P_{x x}(z)+P_{y y}(z)+P_{z z}(z)\right]$.

(a) Stress: The stress is determined from the components of the pressure tensor, and is defined as 


$$
S(z)=P_{z z}(z)-\frac{1}{2}\left[P_{x x}(z)+P_{y y}(z)\right] .
$$

This quantity is interesting for two reasons. First, calculation of the crystal stress is required to determine initial parameters of the interfacial system for an equilibrium interface between fluid and unstrained crystal. Second, the stress profile in the interfacial region provides information about the packing conditions in different parts of the interface.

(b) Orientational order: In order to investigate the type of ordering at the interface we compute the orientational order profile as a function of $z$ on the uniform coarse scale. We quantify "bond" -angle orientational order by the following order parameter:

$q_{n}(z)=\left\langle\frac{1}{N_{z}} \sum_{i, j, k} \cos \left[n \theta_{x y}(i, j, k)\right]\right\rangle$,

where $n$ is an integer number, $i, j$, and $k$ are nearest neighbor atoms in the same $z$ bin, and $\theta_{x y}(i, j, k)$ is the angle between $\mathbf{r}_{i j}$ and $\mathbf{r}_{i k}$ projected on the $x y$ plane. We study $q_{4}(z)$ and $q_{6}(z)$ for both (100) and (111) interfaces, since they best reflect the symmetry of these two orientations.

In order to get information about the extent of the interfacial region for different parameters, we use the so-called 10-90 width, which is defined as the distance over which a monotonically varying parameter profile changes from $10 \%$ to $90 \%$ of its value in the bulk crystal relative to its value in the fluid as one traverses the interface from the fluid into the crystal.

To monitor the stability and determine the coexistence parameters of the crystal-melt interface system, we also compute the crystal and fluid bulk properties. These values are obtained from the parameter profiles by averaging over approximately one-third of the coarse-scaled bins in the middle of either crystal or fluid bulk phase.

Since the density profile at the interface is not known $a$ priori, it is not possible to precalculate the system parameters for a stable interface, even though the coexistence densities for the hard sphere solid and fluid phases are known. Therefore, one usually creates a system fairly close to coexistence and then expects it to equilibrate by itself. However, in the crystal-fluid interface system, because of the periodic boundary conditions, the crystal phase can only adjust its coexistence density by changing the interlayer spacing in the direction perpendicular to the interface. This usually leads to a nonzero stress and strain in the solid phase. In order to construct a truly equilibrated interface, we prepared several trial systems with different initial parameters. Based on the simulation results for these systems, the initial parameters of the system were adjusted so that after equilibration, the crystal in the crystal-fluid interface system remains unstrained.

After the initial parameters for the crystal-melt interface system were determined for both (100) and (111) orientations, we prepared six systems for each orientation with identical initial parameters but different starting configurations and velocities. All 12 systems were allowed to evolve for $20000 \mathrm{cpp}$, which is approximately $550\left(m \sigma^{2} / k_{B} T\right)^{1 / 2}$.
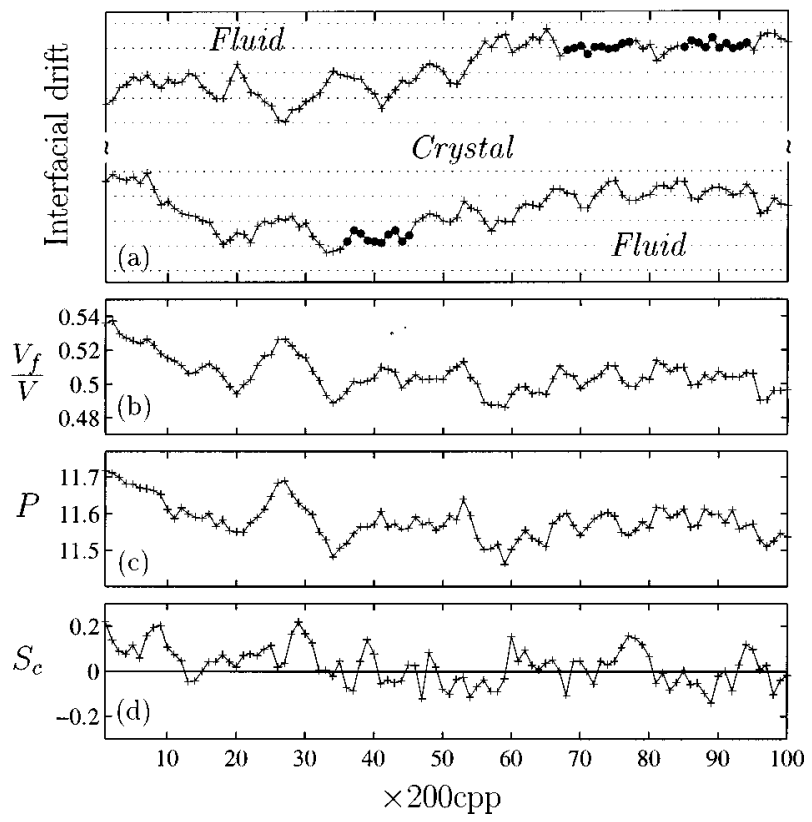

FIG. 1. Drift of the interfaces and time evolution of several system parameters during the simulation run. Each data point represents average quantity over 200 collisions per particle (cpp). (a) Positions of the two crystal-melt interfaces. Distance between horizontal grid lines is equal to the separation between crystal layers. Solid dots represent the intervals selected for the final average. (b) Ratio of volume occupied by the fluid system volume, determined by the distance between the two interfaces, to the total system volume. (c) System pressure. Note that the pressure fluctuations closely follow change in the fluid volume, indicating that they are primarily caused by the freezing and melting at the interfaces. (d) Average crystal stress $S_{c}$ computed from the stress profile inside the crystal. The initial system parameters are chosen such that $S_{c}$ fluctuates around zero during the simulation run.

This corresponds to about $0.4 \mathrm{~ns}$ for a simple fluid such as argon. The interfacial diagnostics were recorded every 200 cpp.

Initially the total pressure in the systems was approximately $11.7 k_{B} T / \sigma^{3}$ with an average positive stress in the bulk crystal of about $0.1 k_{B} T / \sigma^{3}$. After $2000-3000 \mathrm{cpp}$, due to freezing of a portion of fluid next to the interface, the pressure dropped to about $11.5 k_{B} T / \sigma^{3}$ and the bulk crystal became unstrained. However, the magnitude of the pressure fluctuations in individual systems remained large through the whole simulation run, with pressure variations in the range from 11.4 to $11.7 k_{B} T / \sigma^{3}$. The pressure fluctuation were at least an order of magnitude larger compared to the fluctuations in the bulk fluid or crystal system of the same size and were determined to be inversely proportional to the area of the interface. Besides, the pressure fluctuations closely follow fluctuations in relative volume of the fluid phase [see Figs. 1(b) and 1(c)]. Thus, the cause of these fluctuations is a continual process of partial freezing and melting at the interface. This fact has to be taken into account when the time averages of parameter profiles are computed. Indeed, because of the freezing or melting at the interface, the relative position of the interface changes with time and the features of the interface obtained by averaging the profiles over long time intervals would be unrealistically broadened. In order to avoid this broadening, the average quantities should be com- 


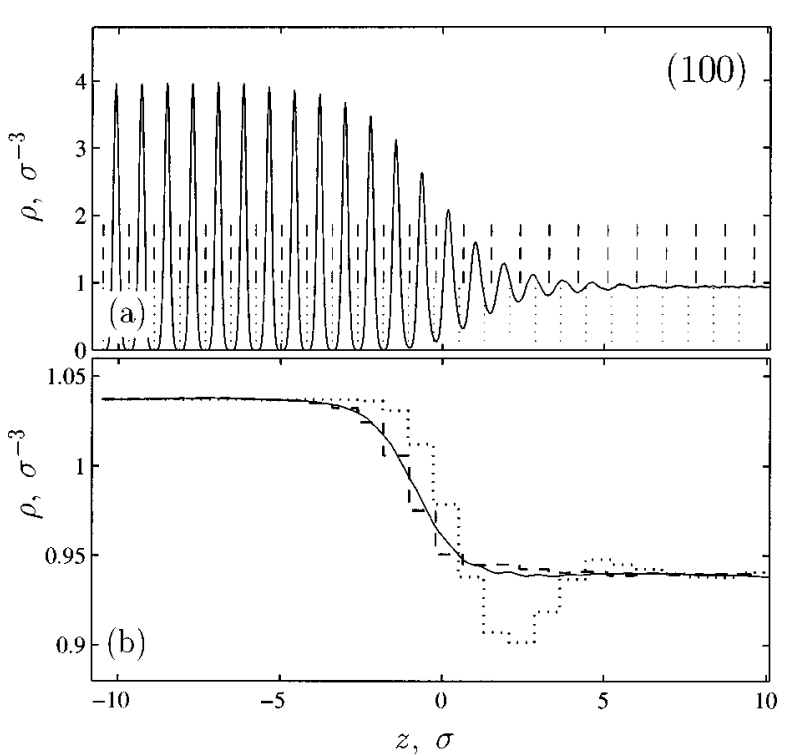

FIG. 2. (a) Fine scale density profiles for the (100) crystal-melt interface. The vertical dotted lines show boundaries of the uniform coarse-scale bins of width equal to the bulk crystal layer spacing. The dashed lines show the nonuniform coarse-scale boundaries placed at the minima of the fine-scale density profiles and extended into the fluid with the spacing between the outmost interface layers. (b) Density profiles on the uniform (dotted line) and the nonuniform (dashed line) coarse scales are shown together with the filtered profile $\bar{\rho}(z)$ (solid line). Here and in subsequent figures, zero on the horizontal axis indicates position of the interface determined from the orientational order parameter profile.

puted only from intervals of the simulation run during which the interface is relatively stationary. In Fig. 1 the time evolution of several parameters for one of the systems and the intervals selected for the computation of averages are shown.

Figure 1(a) shows positions of the interfaces which we determine using the orientational order parameter profiles defined in Eq. (10). We define the position of the interface by the point where the orientational order parameter crosses the average of its values in the bulk crystal and fluid phases. The parameters $q_{4}(z)$ and $q_{6}(z)$ are used to determine positions of the interfaces in (100) and (111) systems, respectively. This method of defining position of an interface was chosen over the more traditional method of determining the Gibbs dividing surface, because the orientational order parameter was found to vary monotonically across the interface with well defined values (relatively small fluctuations) in the bulk crystal and fluid (see Fig. 5 below).

For the final averages we selected intervals of duration $2000 \mathrm{cpp}$ each. The intervals were selected according to two criteria: First, drift of the interface should not exceed half the distance between crystal layers; second, the average crystal stress $S_{c}$ during the selected interval should be smaller than $0.05 k_{B} T / \sigma^{3}$ [see Fig. 1(d)]. The number of thus selected intervals was 16 for each of (100) and (111) orientations.

\section{STRUCTURE}

\section{A. Density profiles}

Density profiles for the (100) and (111) crystal-melt interfaces are shown in Figs. 2 and 3, respectively. The fine-

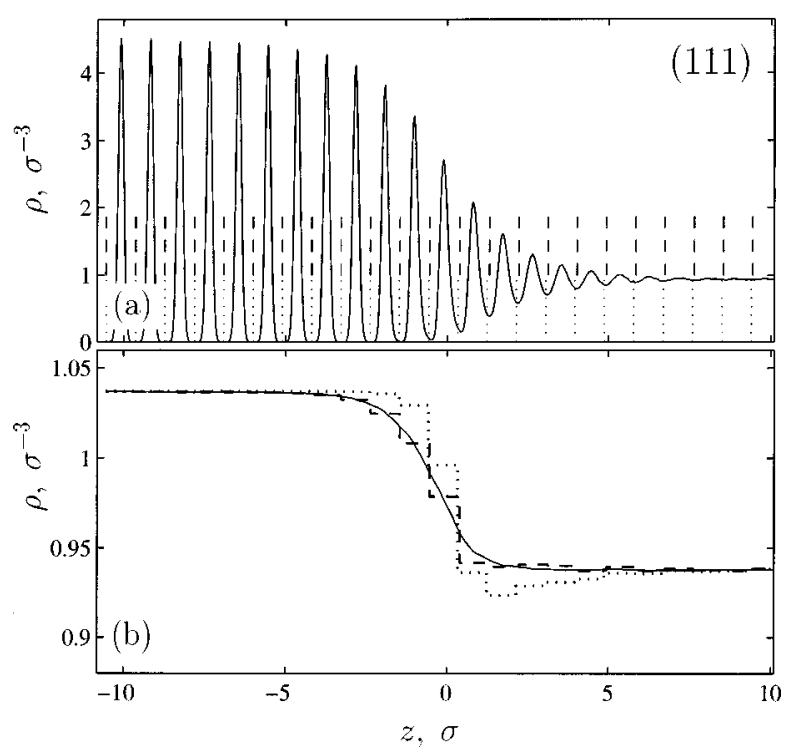

FIG. 3. Fine and coarse-scale density profiles for the (111) crystal-melt interface. (See caption to Fig. 2.)

scale density profiles show large oscillations (corresponding to the crystal layers) that dampen gradually in the interfacial region. The 10-90 widths of the height of the density peaks are about 5.4 and $5.9 \sigma$ for the (100) and (111) interfaces, respectively - these values are consistent with both the $\mathrm{KB}$ and MMN simulations, but the anisotropy between the (100) and (111) widths that we note are more consistent with the KB results, since the MMN widths do not depend measurably on orientation. Note that the maximum density values in the crystal are $4.0 \sigma^{-3}$ and $4.6 \sigma^{-3}$ for (100) and (111) orientations, respectively, which agrees with the results of the KB Monte Carlo simulation. ${ }^{11}$ In the MMN simulation ${ }^{12}$ the corresponding values are $3.2 \sigma^{-3}$ and $3.7 \sigma^{-3}$. This discrepancy is due to the fact that in out simulation the bins are required to move together with the average positions of the crystal layers, which eliminates artificial broadening of the crystal density peaks caused by the drift of the average crystal position with respect to the simulation cell.

Coarse scale density profiles reveal additional information about the structure of the interfaces. However, because of the large oscillations in the crystal density, the appearance of coarse-scaled profiles is extremely sensitive to the way the coarse-scale bins are positioned. This is evident when we plot density profiles on both uniform and nonuniform coarse scales in Figs. 2(b) and 3(b).

The uniform coarse-scale density profile for the (100) interface exhibits pronounced density deficit. ${ }^{16}$ However, when we plot density profile on a nonuniform coarse scale commensurate with the density oscillations, the density deficit disappears. Note that the density on this scale changes from crystal to fluid value in a narrow region of only 4-5 crystal layers. A similar narrow region of the density variation across the interface is seen in the filtered density profiles $\bar{p}(z)$ defined according to Eqs. (1) and (3) with $\epsilon=22.1$ and 22.0 for the (100) and (111) orientations, respectively. The 10-90 widths of the filtered density profiles are 3.2 and $3.3 \sigma$ 
for the (100) and (111) interfaces, respectively. The oscillations in the density profile on the fluid side of the interface seem to occur at essentially constant fluid coexistence density. This phenomenon is exhibited in both (100) and (111) orientations.

It is useful at this point to compare the density profiles produced in the current simulation to those predicted by recent density-functional theory calculations on this same system. In a density-functional theory, information about correlation functions in the bulk fluid phase is used to construct the Helmlholtz free-energy functional $\mathscr{F}[\rho(\mathbf{r}]$, where $\rho(\mathbf{r})$ is the usual single-particle density. An interface calculation consists then of parameterizing $\rho(\mathbf{r})$ such that one obtains the coexisting bulk crystal and bulk liquid phases far from the interfacial region and minimizing the surface free energy (which is calculated from the functional for $\mathscr{F}$ with respect to the parameters. (For more details on the types of parameterizations and functional approximation that have been used, see the reviews cited in the first paragraph of the Introduction.) By far the most ambitious such calculation is that of Ohnesorge et al. ${ }^{17}$ the weighted-density approximation of Curtin and Ashcroft ${ }^{18}$ is used together with a nearly free parameterization of the density. The 10-90 width for their calculated density profiles (fine scale) are 2.6 and $2.9 \sigma$ for the (100) and (111) hard-sphere interfaces, respectively. These are considerably smaller than those found here. The DFT calculations also show little expansion (less that 2\%) of the peak spacing in the (100) interface, which is clearly in contradiction to this and earlier simulations. The source of the difference between the simulation and the theory is not clear. One possible origin is the mean-field nature of the density-functional calculation which cannot properly take into account the inhomogeneity of the interfacial region where the simulations show coexisting clusters of solid and liquid nature. Kyrlidis and Brown ${ }^{11}$ have also recently used DFT to calculate the hard-sphere solid-liquid interfacial structure. The functional that they used is based on the generalized effective liquid approximation (GELA) of Lutsko and Baus, ${ }^{19}$ which, for hard spheres (and hard spheres only!), gives more accurate results for the coexistence densities than does WDA. Using a parameterization that was less general than that of Ohnesorge et al., their 10-90 widths for the density profiles were 1.6 and $1.5 \sigma$ for (100) and (111), respectively-narrower than the results of Ohnesorge et al., and much narrower than the present simulation results.

\section{B. Interlayer separation}

To study changes in the crystal layer spacing across the interface, we define interlayer separation

$$
\Delta z_{i}=\bar{z}_{i+1}-\bar{z}_{i},
$$

where $\bar{z}_{i}$ is the center of mass of layer $i$ determined from the fine-scale density profile between the adjoining density minima. Figure 4 shows a large difference between the (100) and (111) interface orientations. The (100) layers show a very large expansion from $0.784 \sigma$ in the crystal to $0.901 \sigma$ between the last two detectable layers on the fluid side of the interface. The (111) layers, however, exhibit a small expansion from $0.906 \sigma$ in the crystal to $0.926 \sigma$ in the middle of

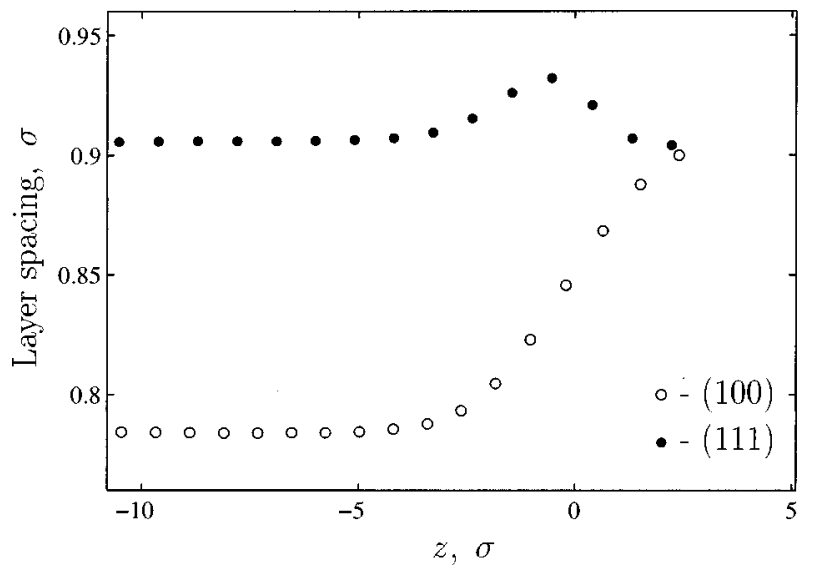

FIG. 4. Layer separation for (100) and (111) interfaces.

the interface, followed by a contraction to slightly below the interlayer spacing in the bulk crystal. Very similar results for the layer width variations (including the small expansion of the (111) interface) were reported for a 35000 particle MD simulation of the Lennard-Jones crystal-melt interface by Galejs, Raveche, and Lie. ${ }^{20}$ Obviously, the repulsive part of the Lennard-Jones potential is a major factor determining the structure of the crystal-melt interfaces in those systems.

\section{Orientational order profiles}

The fact that the interlayer spacing for the (100) interface increases at the interface and approaches the width of the (111) crystal layers was speculated in earlier work to indicate a preference of the fluid to order at a planar interface in a way that is more consistent with a (111) face. ${ }^{11}$ We test this conjecture by calculating the orientational order parameter profiles $q_{4}(z)$ and $q_{6}(z)$ as defined in Eq. (10). The (111) ordering in the (100) interface should then exhibit itself in a nonmonotonic behavior of the $q_{6}(z)$ profile with a maximum in the interfacial region. The results, presented in Fig. 5, suggest that no ordering of the (100) interface consistent with the (111) symmetry is present, since all the profiles change monotonically across the interface. The 10-90 widths for all of the orientation profiles vary between 3.0 and $3.0 \sigma$.

Relative widths and positions of the fine and coarsescale density profiles as well as the difference in the interlayer spacings between the (100) and (111) orientations seem to be consistent with the interpretation of the interfacial structure given by Broughton and Gilmer, ${ }^{21}$ who have done extensive molecular dynamics simulations of the LennardJones crystal-melt interface. They attribute the density variations on the fluid side of the interface to the properties of the structure factor $S(q)$ of the homogeneous fluid at the coexistence density. The density variations induced in the fluid are most likely to have a wavelength consistent with the position of the maximum of the fluid structure factor. Since this wavelength has a value close to the (111) crystal layer separation, the (111) interfacial layers do not significantly change their width, while the (100) layers tend to relax outwards. Note that the interfacial region defined in the orien- 


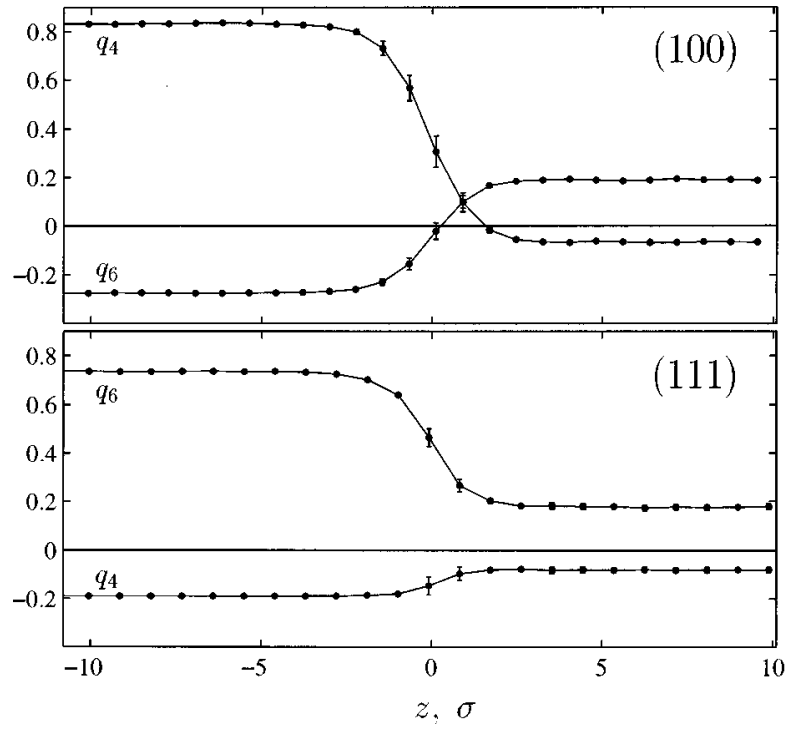

FIG. 5. Orientational order parameter profiles measured on a uniform coarse-scale according to Eq. (10). Error bars show twice the standard deviation of the mean in every bin computed from the 16 selected intervals.

tational profiles and the coarse-scale density profiles have nearly idential widths and positions-the significance of this is discussed in the summary section.

\section{In-plane density distributions}

In addition to the $z$-dependent profiles, we monitor the in-plane 2-D density distribution at the interface by dividing each uniform coarse-scale bin into an array of cells in the $x y$ plane. The array size was $80 \times 80$ for the $(100)$ and $78 \times 86$ for the (111) orientation. For comparison we pick one bin in the bulk crystal, one bin in the fluid, and four adjacent bins in the middle of the interface. The resulting $x y$-plane density distributions for (100) and (111) orientations are shown in Figs. 6 and 7, respectively, together with the fine-scale density profiles with the selected coarse-scale bins indicated by letters $\mathbf{A}$ through $\mathbf{F}$. The density distributions are produced by averaging over $2000 \mathrm{cpp}$ for one of the systems in each orientation.

The plots show that for both orientations the transition from a crystal-like to a fluidlike structure occurs over a narrow region of only a couple of layers. The layer $\mathbf{B}$ has a perfectly ordered structure similar to the bulk crystal layer $\mathbf{A}$, except with broader density peaks located at the crystal lattice sites. On the other hand, the layer $\mathbf{E}$ looks very much like the bulk fluid layer $\mathbf{F}$. Note that the fluid layer density is not very uniform, since the time interval, over which the density distribution is monitored, is not sufficiently long to get the local density fluctuations averaged over.

Another feature one can clearly see is that within each interfacial layer there are ordered and disordered regions. This is indicative of a rough, inhomogeneous interface. The coexistence of ordered and disordered regions within interfacial layers was reported in earlier studies of crystal-melt interfaces of simple liquids. ${ }^{20,22,23}$ However, an important issue, which was not fully addressed in the previous studies, is

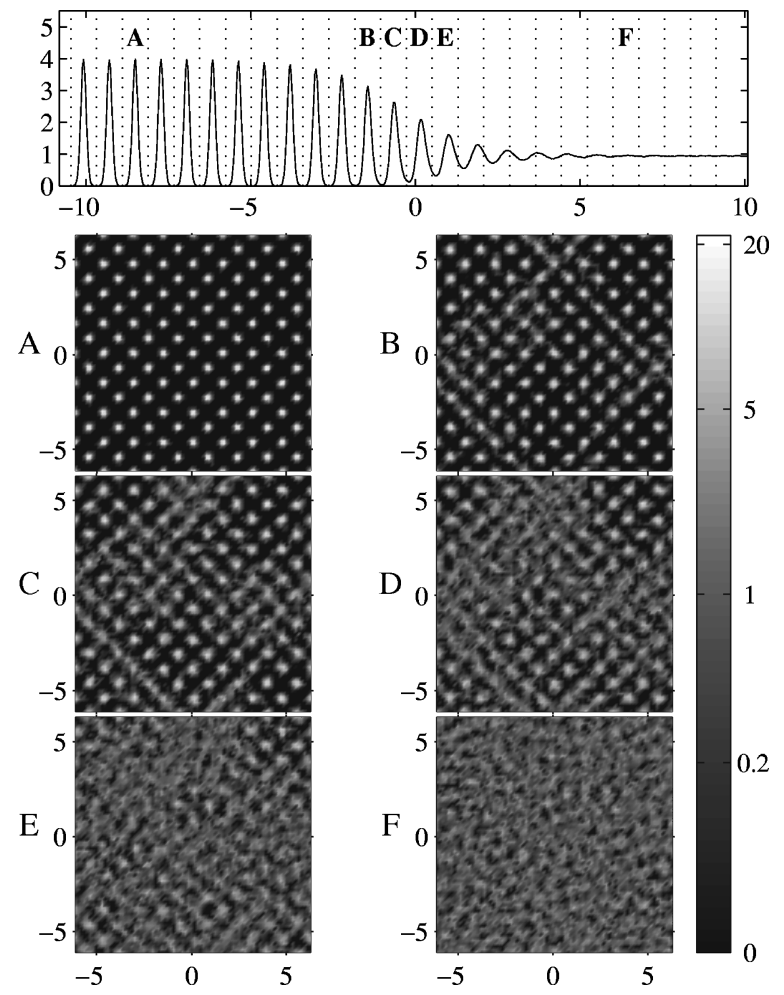

FIG. 6. Density variations in the $x y$ plane for different layers of the (100) interface. The narrow vertical bar shows the gray scale corresponding to different density values.

the dependence of the interface roughness on the time scale over which the interface is monitored. Our simulations show that when averaged over long time intervals (10 $000 \mathrm{cpp}$ and more), the in-plane density distributions become much more

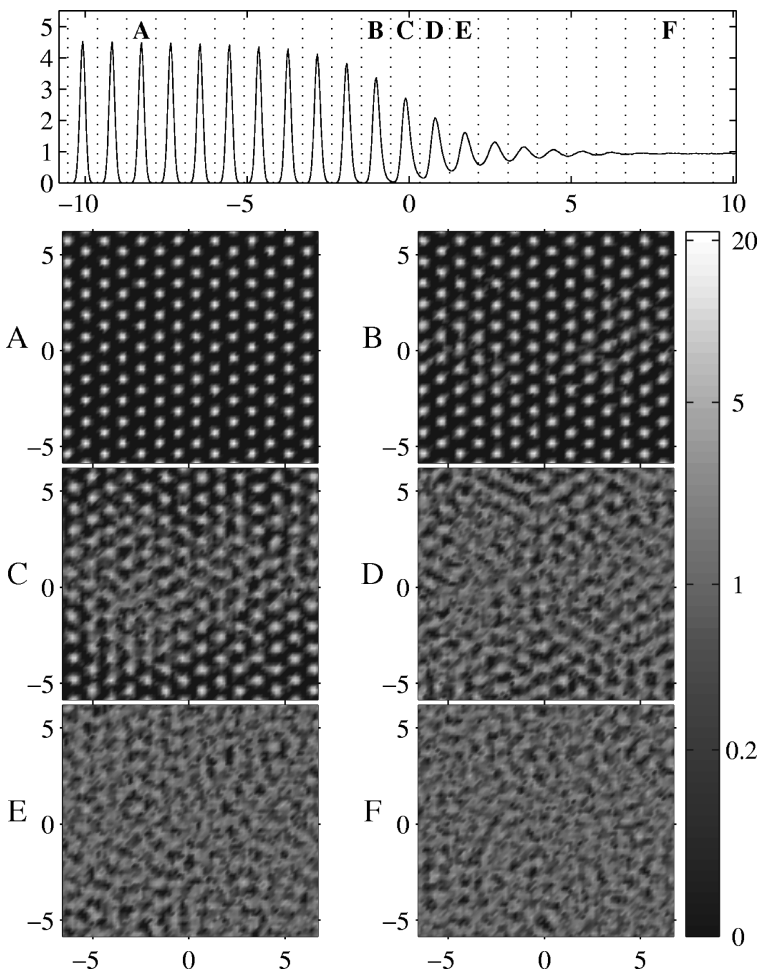

FIG. 7. Density variations in the $x y$ plane for different layers of the (111) interface. 


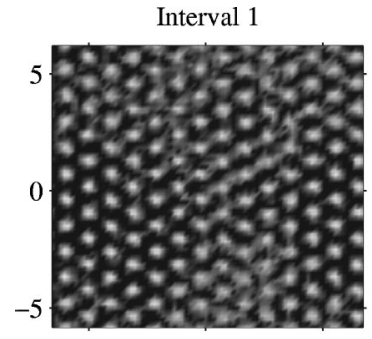

Interval 3
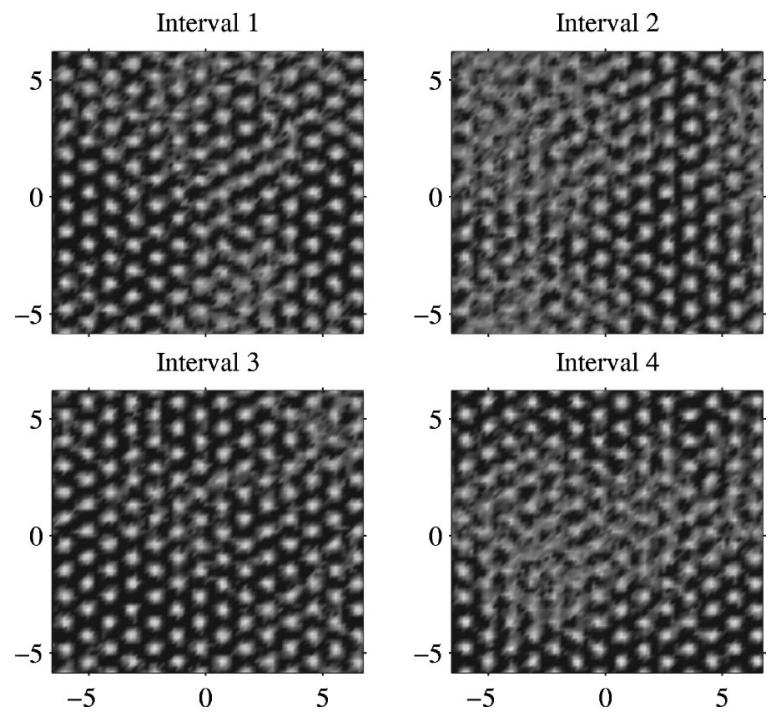

Interval 4

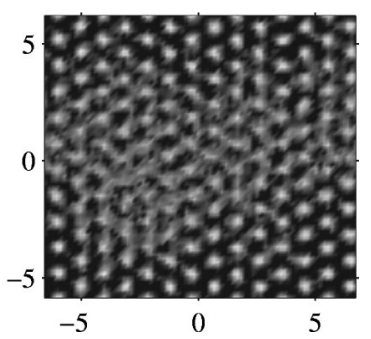

FIG. 8. Density distributions in the layer $\mathbf{C}$ of the (111) interface obtained by averaging over four consecutive time intervals of $2000 \mathrm{cpp}$ duration each.

uniform. This can be explained by a high mobility of the ordered and disordered regions.

As an example we show in Fig. 8 four snapshots of the density distribution in the layer $\mathbf{C}$ of the (111) interface obtained by averaging over four consecutive time intervals each of $2000 \mathrm{cpp}$ duration. Each snapshot shows a different arrangement of the ordered and disordered regions. Therefore, averaged over the four intervals, the density distribution would appear to have less roughness than the individual snapshots. As a consequence, the longer the time interval over which the interface is monitored, the more diffuse and homogeneous it appears.

\section{PRESSURE AND STRESS PROFILES}

The pressure profiles are measured on the fine scale according to Eqs. (6) and (7). From these we calculate the filtered transverse pressure profile $\bar{P}_{z z}(z)$ and the filtered stress profile $\bar{S}(z)$, which are shown in Figs. 9 and 10, respectively. The transverse pressure profiles are uniform across the interface, which is an indication that the interfaces are in mechanical equilibrium. The stress profile shows no statistically significant stress or strain in the bulk crystal and the profile, as expected, is not uniform through the interface.

We have also tried to determine the coarse-scale pressure profiles using a nonuniform binning procedure, but have found that they cannot be defined in a consistent manner. The nonuniform coarse-scale profiles appear similar to the filtered profiles, but the nonuniform bin boundaries have to be determined separately for each profile according to its own minima. This makes it hard to define consistently the total pressure and stress profiles. On the other hand, when we attempt to use the same nonuniform bin boundaries for all the profiles, we obtain profiles with features which appear to be the artifacts of particular positions of bin boundaries. For example, when the nonuniform bin boundaries are determined according to the minima of the fine-scale density profiles as in Figs. 2 and 3, the transverse pressure profiles ex-

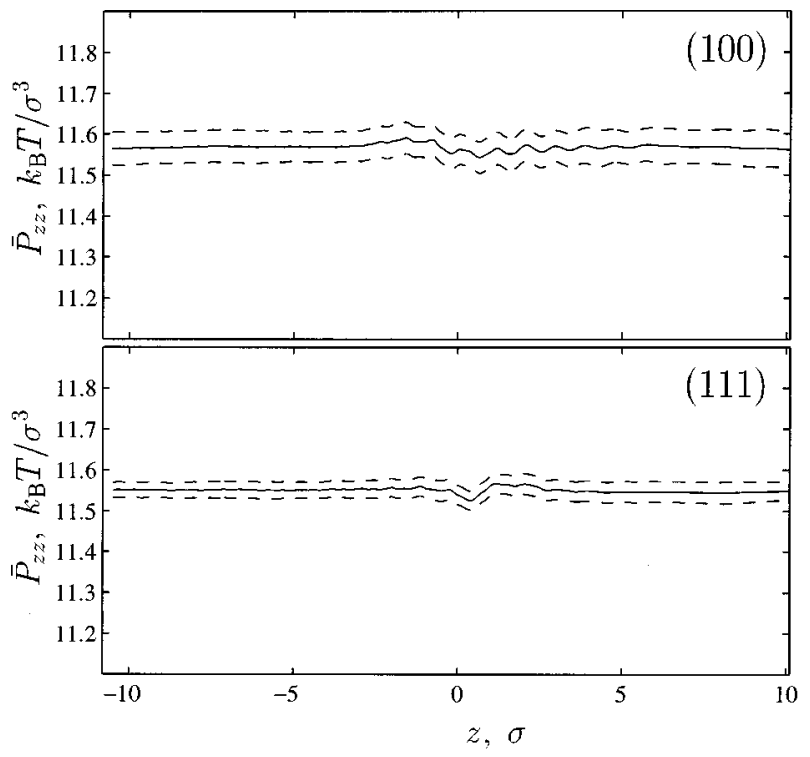

FIG. 9. Filtered transverse pressure profiles for the (100) and (111) interfaces. The dashed lines show twice the standard deviation of the mean pressure in every bin computed from the 16 selected intervals.

hibit nonuniform features in the interfacial region, which can be removed by adjusting the bin boundaries according to the minima of the fine-scale transverse pressure profiles. These problems illustrate the superiority of the FIR filtering techniques over the usual binning process for defining coarsegrained averages of oscillatory quantities.

The stress profiles for the two orientations are shown in Fig. 10. The surface stress can, in theory, be obtained from the area under the stress profile $S(z)^{2}$. Integrating over the interfacial region between $-5 \sigma$ and $5 \sigma$ we obtain the surface stress $-0.17 \pm 0.06 k_{B} T / \sigma^{2}$ for the (100) interface and $-0.71 \pm 0.13 k_{B} T / \sigma^{2}$ for $(111)$.

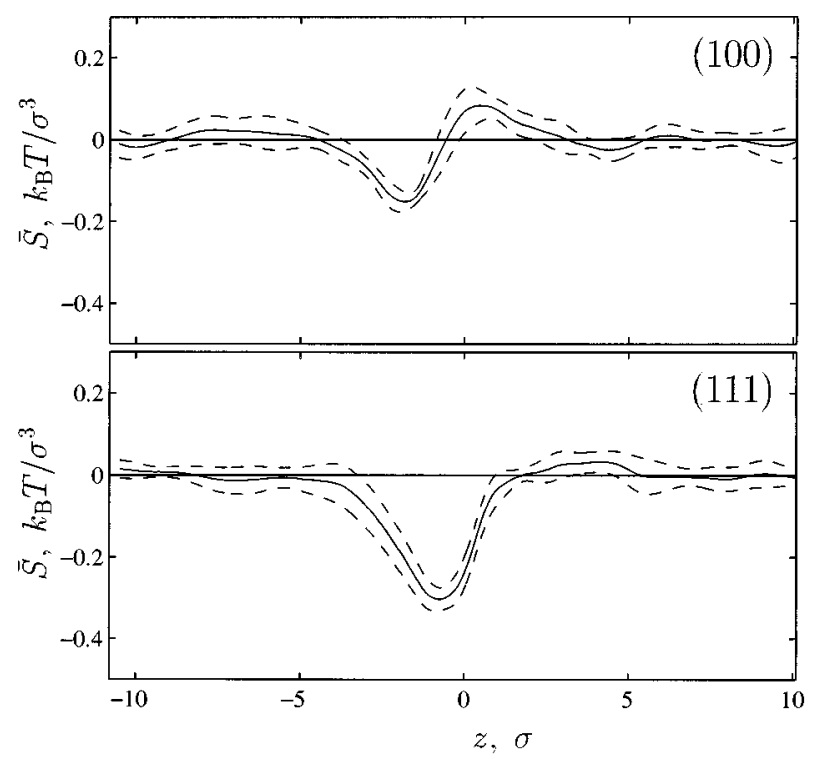

FIG. 10. Stress profiles for the (100) and (111) interfaces. The dashed lines show twice the standard deviation of the mean value in every bin computed from the 16 selected intervals. 


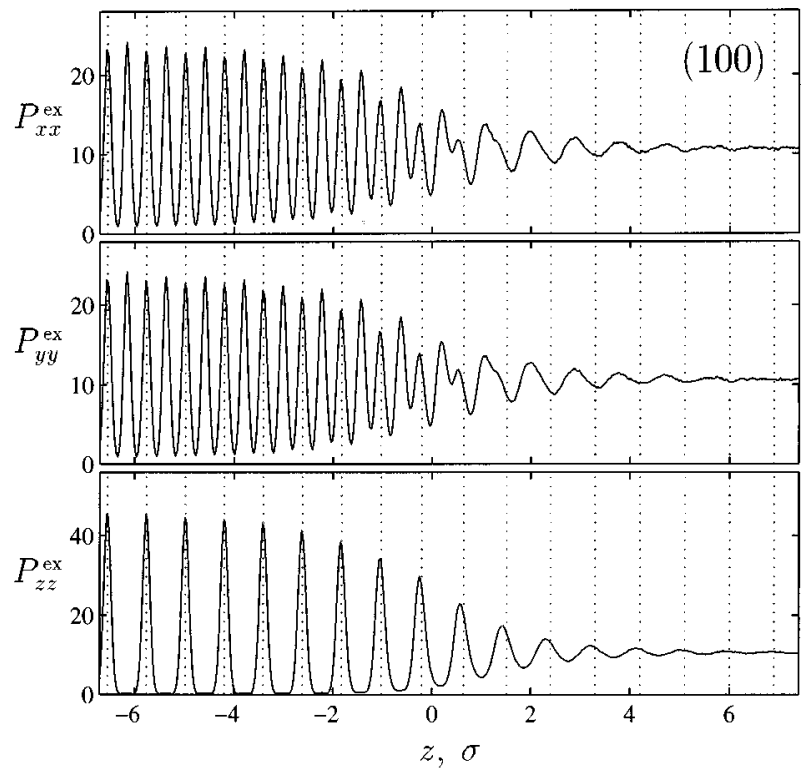

FIG. 11. Excess pressure tensor components on a fine scale for the (100) interface. Vertical dotted lines indicate the nonuniform coarse-scale bin boundaries. Note different scale for the $P_{z z}^{\mathrm{ex}}$ component.

It is also interesting to study the excess pressure on a fine scale, especially if we can distinguish between the pressure contributions from the in-plane collisions and those from the collisions of particles in different crystal layers. To achieve this we modify the binning procedure for the excess pressure measurement. Instead of selecting the bins based on the positions of the centers of the spheres, we select them based on the position of the point of contact between the colliding spheres. Now the excess pressure profile in the crystal will have distinct peaks for the in-plane and interplane collisions. The results are shown in Figs. 11 and 12 for the (100) and (111) interfaces, respectively.

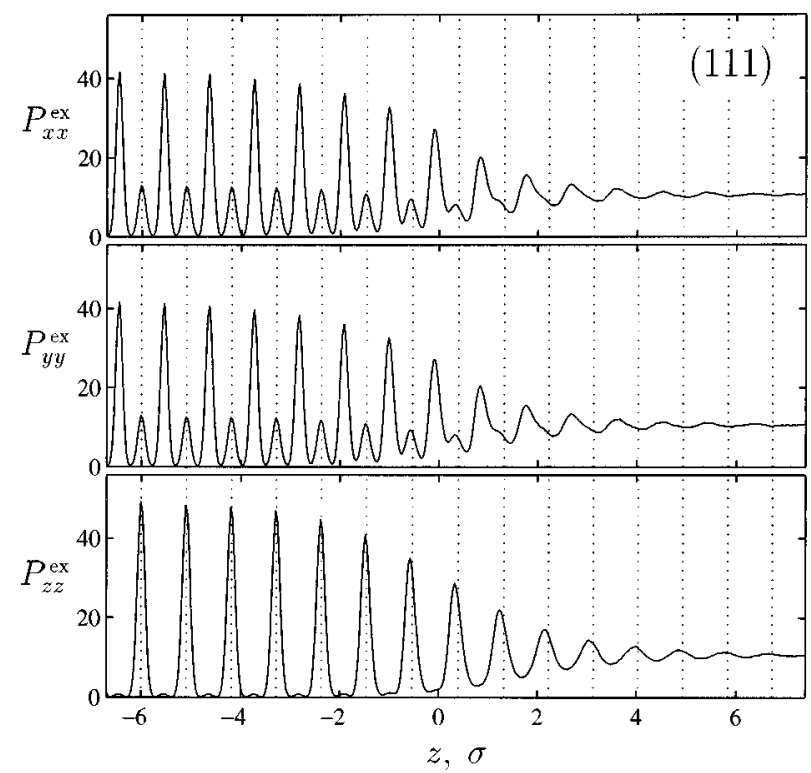

FIG. 12. Excess pressure tensor components on a fine scale for the (111) interface.
For both the (100) and (111) orientations the $P_{z z}^{\mathrm{ex}}(z z)$ profiles have peaks corresponding only to the interplane collisions, because there is very little average momentum transfer in the $z$ direction during the in-plane collisions. On the other hand, the $x x$ and $y y$ components are alike and have peaks both within and between the crystal layers. Difference in the peak height for the (111) interface reflects the tighter packing within crystal layers for this orientation. When the interface is traversed from crystal into fluid, the in-plane and interplane peaks of the $x x$ and $y y$ components decay and blend together, with the in-plane peaks decaying slower. These profiles are helpful in distinguishing the crystal layered structure from the disordered fluid, exhibiting some residual inhomogeneity. The $z z$ component oscillations decay in the same manner as the number density profile oscillations, except that they are shifted with respect to each other by a half of the crystal layer width.

\section{DYNAMICS}

\section{A. Diffusion coefficient profiles}

Mass transport in the interface is best quantified through the diffusion constant profile $D(z)$, which is important in the understanding of near-equilibrium crystal growth. The diffusion constant was determined from the slope of the mean squared displacement as a function of time

$$
D(z)=\frac{1}{6 N(z)} \frac{d}{d t} \sum_{j=1}^{N(z)}\left\langle\left[\mathbf{r}_{j}(t)-\mathbf{r}_{j}\left(t_{0}\right)\right]^{2}\right\rangle,
$$

where $N(z)$ is the number of spheres between $z-\Delta z / 2$ and $z+\Delta z / 2$ at time $t=t_{0}$, and the brackets represent the average over time origins $t_{0}$. The sphere displacement was monitored on a uniform coarse scale over time $t_{\max }-t_{0}$ $=5.5\left(m \sigma^{2} / k_{B} T\right)^{1 / 2}$ During this time the average fluid particle displacement is less than one particle diameter, so this bin assignments should remain valid for the entire process. The averaging was done over 50 time origins for each of the 16 selected intervals. The average diffusion constant profiles for the (100) and (111) interfaces are shown in Fig. 13. The average diffusion constant in the bulk fluid is $0.024\left(k_{B} T \sigma^{2} / m\right)^{1 / 2}$, which corresponds to about $0.0015 \mathrm{~cm}^{2} / \mathrm{s}$ for a simple liquid such as argon. The diffusion profiles are similar for the (100) and (111) orientations with the 10-90 widths of $3.1 \sigma$ and $3.2 \sigma$, respectively. These widths are nearly identical to the widths obtained from the coarse-grained density profiles. As a test of the diffusion anisotropy, the $x, y$, and $z$ components of the mean square displacement were calculated separately, but were found not to differ significantly within the simulation error. As far as we know, there is no current theory suitable for the quantitative prediction of the diffusion profiles.

\section{B. Vacancy propagation}

Studying density profiles on a uniform coarse scale enabled us to observe the creation and propagation of vacancies from the interface into the bulk crystal. Indeed, the uniform coarse-scale density in the crystal is exactly proportional to the number of particles in each crystal layer, unless there is a 


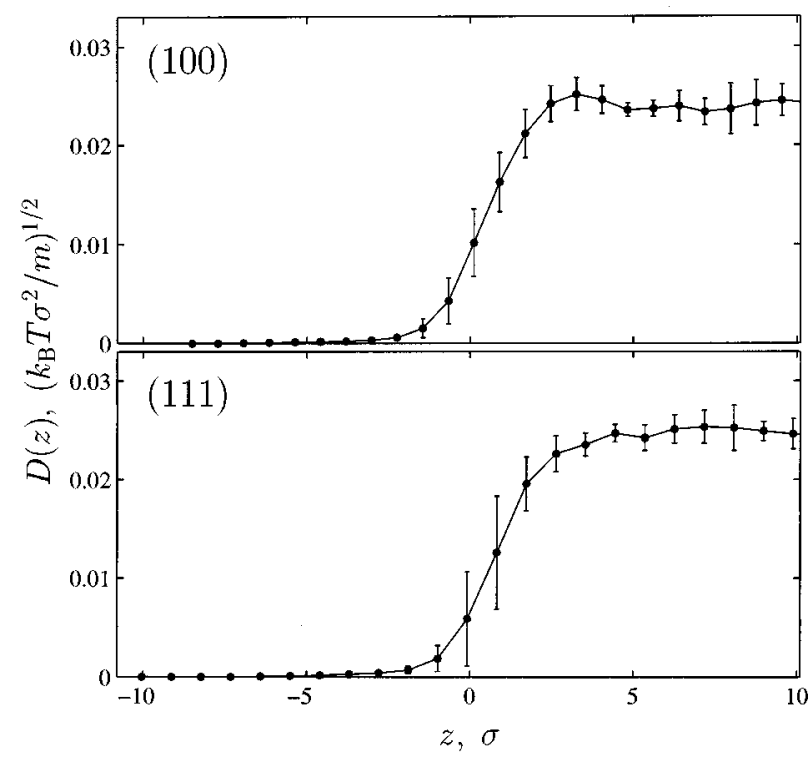

FIG. 13. Diffusion coefficients for the (100) and (111) crystal-melt interfaces. The error bars represent twice the standard deviation of the mean value calculated from the 16 samples.

vacancy, which can be clearly seen as a dip in the density profile. In Fig. 14 the vacancy propagation in one of the (111) simulation runs is shown. Vacancy propagation occured five times in the (100) and four times in the (111) interface systems. (Note that, in this particular case, the vacancy creation coincides with the growth of the left interface by one crystal layer. However, as this coincidence does not appear in many of the other runs in which vacancies were produced, no correlation between vacancy formation and interfacial growth can be inferred from the present data.) The presence of vacancies in the crystal did not have any noticeable effect on the fine-scale density profiles or any other

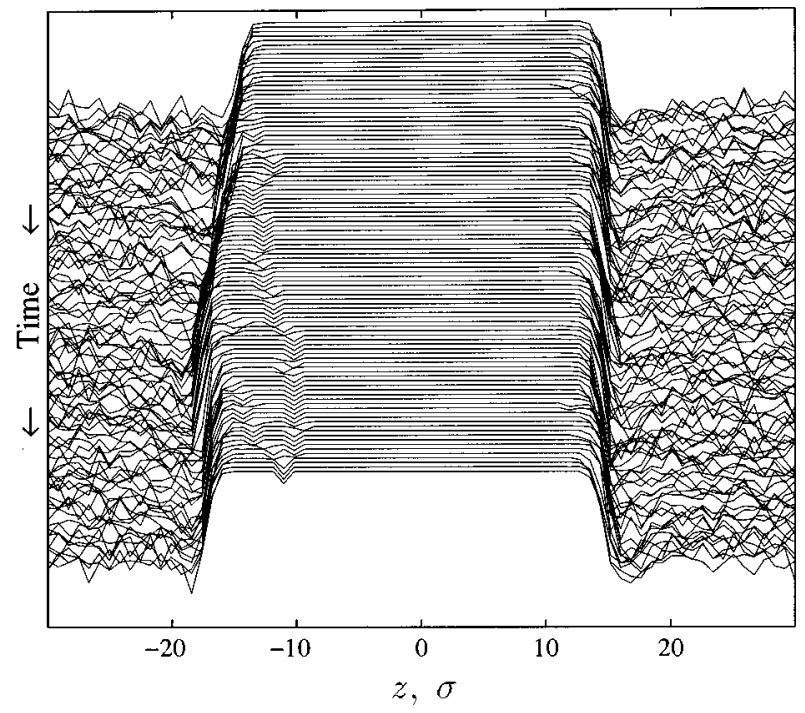

FIG. 14. Vacancy propagation into the (111) crystal. The uniform coarsescale density profiles for one of the simulation runs are shown. The profiles are computed from 100 successive time intervals of $200 \mathrm{cpp}$ duration each, and are shifted downward by $0.005 \sigma^{-3}$ from each other, so that lower profiles correspond to later simulation times. interface characteristics. The mechanism of vacancy formation and a quantitative analysis of the motion of the vacancies in the bulk were not studied here, but will be the subject of future work.

\section{SUMMARY AND CONCLUSION}

We have presented detailed molecular dynamics simulation results for the (100) and (111) FCC crystal-melt interfaces for the single-component hard sphere system. The principal findings of this study can be summarized as follows:

(1) In agreement with previous studies of both the hardsphere interfaces ${ }^{11,12}$ and those for a Lennard-Jones system, ${ }^{20,21}$ we see an increase in the spacing between the (100) density profile peaks as the interface is traversed from solid to liquid. A similar increase is not seen in the (111) interface (the spacing for this interface does increase slightly in the interfacial region, but decreases back to nearly its original value before the density oscillations damp out in the liquid). Analysis of the orientational order in the planes perpendicular to the interface shows that this phenomenon is not due to a (111)-like ordering of the liquid near the (100) interface, as has been previously speculated, ${ }^{11}$ but is more likely due to the properties of the fluid structure factor $S(q)$ - density variations in the fluid that are induced by the presence of the interface will be enhanced if the wavelength is consistent with the position of the first maximum of $S(q){ }^{21}$

(2) We have calculated interfacial profiles of density, pressure and stress using both fine and coarse scales. The coarse-scale profiles for such properties, for which the fine-scale profile is oscillatory, are found to be very sensitive to the process by which they are calculated. Use of uniformly spaced bins is shown to lead to structural artifacts in the interfacial region if the spacing between the oscillation peaks on the fine scale is not constant-as is the case for the (100) interface [and to a much smaller degree (111)]. The use of nonuniformly spaced bins that are commensurate with the oscillations does eliminate the artifacts, but the precise bin spacings needed are shown to be dependent on the property measured, e.g., nonuniform bin spacings optimized for density profiles will lead to structural artifacts if used to calculate a coarse-grained pressure profile. As a way around these problems, we demonstrate that well-defined coarsegrained profiles can be produced from the fine-scale profiles without the use of an additional binning procedure through the use of a finite impulse response (FIR) filter. $^{14}$

(3) Analysis of density contours in the $x-y$ planes perpendicular to the interface show that the actual transition from crystal to fluid takes place over a relatively narrow region-about 2-3 crystal layers. This is much narrower than the width indicated by the density profiles, which are 7 to 8 crystal planes in width. Also, the transition layers are not uniform, but exhibit domains of crystal and fluid that coexist within the same layer. These domains are not static, but appear to have a high degree of mobility-even on short time scale (subnanosecond) in- 
herent in such computer simulations. This clustering could prove problematic for the construction of an accurate density-functional theory of the solid-fluid interface since such theories are mean-field in nature and will not include the contributions of such dynamic fluctuations.

(4) The diffusion constant profiles for each interface have been calculated. The interfacial widths indicated by this transport property are independent of interfacial orientation and are about 3.1 to $3.2 \sigma$, which corresponds to about four (100) lattice planes and three (111) planes. These widths are nearly identical to the widths obtained from the coarse-grained density profiles (3.2 to $3.3 \sigma$ ), but are narrower than the widths of fine-grained density peaks which vary from 5.4 to $5.9 \sigma$. The behavior of the diffusion constant is nearly identical to that seen in previous interfacial simulations on Lennard-Jones and inverse power potentials. ${ }^{24}$

(5) We show that the interfacial region defined by coarsescale density profiles and the orientation profiles have nearly identical width and position. These quantities are measures of the "intrinsic" width of the interface, that is, the distance over which the system goes from being solidlike to being liquidlike. This width is significantly smaller (and more isotropic) than the structural width of the interface (defined as the width of the fine-scale density profiles). This difference is due to the fact that the crystal lattice peaks begin to broaden before any significant liquid disorder begins to emerge and, on the liquid side, the liquid begins to exhibit $z$ direction ordering before any solidlike $x y$ ordering sets in. This is also indicated in the $x y$-plane density contours. It is interesting to note that the diffusion profiles have the same width as both the coarse-scale density profiles and the orientation profiles, but the position of the apparent interface is shifted considerable towards the liquid phase.

(6) We have observed in some of our simulation runs the creation and propagation of vacancies from the interface into the bulk crystal. The precise creation mechanism and transport properties of these vacancies were not investigated in this study.

\section{ACKNOWLEDGMENTS}

This research was supported by National Science Foundation Grant No. CHE-950281. The authors also thank the Kansas Institute for Theory and Computational Science (KITCS) for providing a stimulating research environment as well as the Kansas Institute for Advanced Scientific Computing (KCASC) for the use of their computational facilities for the completion of some of the work herein.

${ }^{1}$ D. P. Woodruff, The Solid-Liquid Interface (Cambridge University Press, London, 1973).

${ }^{2}$ W. A. Tiller, The Science of Crystallization: Microscopic Interfacial Phenomena (Cambridge University Press, New York, 1991).

${ }^{3}$ J. M. Howe, Interfaces in Materials (Wiley, New York, 1997).

${ }^{4}$ B. B. Laird and A. D. J. Haymet, Chem. Rev. 92, 1819 (1992).

${ }^{5}$ Some experimental attempts have been made, however-for example, Howe [Philos. Mag. A 74, 761 (1996)], using high-resolution transmission electron microscopy to examine the interface between crystalline $\mathrm{Pd}_{3} \mathrm{Si}$ and amorphous $\mathrm{Pd}_{80} \mathrm{Si}_{20}$, obtained direct experimental evidence that the first several layers of the amorphous phase were ordered at the interface.

${ }^{6}$ Y. Singh, Phys. Rep. 207, 351 (1991).

${ }^{7}$ H. Löwen, Phys. Rep. 237, 249 (1994).

${ }^{8}$ J. Q. Broughton and G. H. Gilmer, J. Chem. Phys. 84, 5759 (1986).

${ }^{9}$ M. A. Allen and D. J. Tildesley, Computer Simulation of Liquids (Oxford Science Press, Oxford, 1987).

${ }^{10}$ J. P. Hansen and I. R. McDonald, Theory of Simple Liquids, 2nd ed. (Academic, New York, 1986).

${ }^{11}$ A. Kyrlidis and R. A. Brown, Phys. Rev. E 51, 5832 (1995).

${ }^{12}$ A. Mori, R. Manabe, and K. Nishioka, Phys. Rev. E 51, R3831 (1995).

${ }^{13}$ W. G. Hoover and F. H. Ree, J. Chem. Phys. 49, 3609 (1968).

${ }^{14}$ W. H. Press, S. A. Teukolsky, W. T. Vetterling, and B. P. Flannery, Numerical Recepies in Fortran, 2nd ed. (Cambridge University Press, Cambridge, 1992).

${ }^{15}$ R. L. Davidchack and B. B. Laird, Phys. Rev. E 54, R5905 (1996).

${ }^{16}$ In an earlier work (Ref. 12), we reported a similar deficit in the binary hard sphere (100) interface with $\sigma_{1} / \sigma_{2}=0.9$ while using uniform bins. Our claim that this deficit was independent of the binning procedure was, in light of the current results, incorrect.

${ }^{17}$ R. Ohnesorge, H. Lowen, and H. Wagner, Phys. Rev. E 50, 4801 (1995).

${ }^{18}$ W. A. Curtin and N. W. Ashcroft, Phys. Rev. A 32, 2909 (1985).

${ }^{19}$ J. F. Lutskso and M. Baus, Phys. Rev. Lett. 64, 761 (1990).

${ }^{20}$ R. J. Galejs, H. J. Raveche, and G. Lie, Phys. Rev. A 39, 2574 (1989).

${ }^{21}$ J. Q. Broughton and G. H. Gilmer, J. Chem. Phys. 84, 5749 (1986).

${ }^{22}$ J. Tallon, Phys. Rev. Lett. 57, 1328 (1986).

${ }^{23}$ O. A. Karim and A. D. J. Haymet, J. Chem. Phys. 89, 6889 (1988).

${ }^{24}$ B. B. Laird and A. D. J. Haymet, J. Chem. Phys. 91, 3638 (1989). 